\title{
Impacto jurídico de la Organización para la Cooperación y el Desarrollo Económico (OCDE) en Colombia ${ }^{1}$
}

\section{Legal impact of the Organization for Economic Co-Operation and Development (OECD) in Colombia}

\author{
DOI: http://dx.doi.org/10.17981/jurideuc.14.1.2018.4
}

Artículo de investigación. Fecha de recepción: 05/02/2018 Fecha de aceptación: 22/08/2018

\author{
Luz Elena Mira Olano \\ Institución Universitaria Tecnológico de Antioquia (Colombia) \\ luz.mira@tdea.edu.co
}

\author{
Jahir Alexander Gutiérrez Ossa \\ Institución Universitaria Tecnológico de Antioquia (Colombia) \\ jagogutierrez@gmail.com
}

Para citar este artículo:

Mira, L. y Gutiérrez, J. (2018). Impacto jurídico de la Organización para la Cooperación y el Desarrollo Económico (OCDE) en Colombia. JURÍDICAS CUC, vol. 14, no. 1, pp. 75-104. DOI: http://dx.doi.org/10.17981/juridcuc.14.1.2018.4

\section{Resumen}

El artículo diagnostica el impacto jurídico proveniente de la adhesión del Estado colombiano a la Organización para la Cooperación y el Desarrollo Económico (OCDE). El denominado "Club de buenas prácticas" pondrá al Estado colombiano en la capacidad de dar cuenta de diferentes capítulos a través de cambios en la legislación con fines de política pública. La simbiosis entre el método descriptivo y explicativo materializan las implicaciones jurídicas planteadas en los 23 capítulos aprobados por la OCDE para Colombia y el componente metodológico depende de la capacidad de respuesta del país frente a ellos. El desarrollo del artículo está enfocado a: i) diagnosticar el impacto jurídico de la OCDE sobre Colombia desde los 23 capítulos aprobados; ii) la capacidad de respuesta del Estado colombiano para incorporar las modificaciones jurídicas planteadas por la OCDE, y iii) el recibimiento que tendría la incorporación de la legislación requerida en los sectores estratégicos del país. Palabras clave: buenas prácticas; comités OCDE; cooperación internacional; desarrollo económico; política pública; regulación internacional.

\begin{abstract}
This paper analyzes the legal impact drawn from the incorporation of Colombia as a member of the Organization for Economic Cooperation and Development (OECD). The so-called "Club of good practices" will grant the Colombian State the opportunity to approach different chapters by making changes in the legislation towards public policymaking. A symbiosis between a descriptive and explanatory method materialize the legal implications set out in the 23 chapters approved by the OECD for Colombia and the methodological component depends on the response capacity of the country to them. The development of this paper is focused on: i) analyzing the legal impact of the OECD in Colombia in terms of the 23 chapters approved; ii) determining the response capacity of the Colombian State to incorporate the legal changes raised by the OECD, and iii) the receipt that would be the incorporation of the legislation required in the strategic sectors of the country.

Keywords: good practices; OECD committees; international cooperation; economic development; public policy; international regulation
\end{abstract}

\footnotetext{
${ }^{1}$ Este artículo es producto del proyecto de investigación: Los alcances de la jurimetría y la estrategia legal en el ordenamiento jurídico colombiano. Realizado por el Grupo de Investigación Jurídica y Social del Programa de Derecho, Facultad de Derecho y Ciencias Forenses, Institución Universitaria Tecnológico de Antioquia.
}

The author; licensee Universidad de la Costa - CUC. 


\section{INTRODUCCIÓN}

Es conocida la trayectoria de la OCDE para los países que hacen parte del selecto grupo de países con "Buenas prácticas o los denominados países ricos". La OCDE desde su creación ha estado orientada a fortalecer el aparato institucional de los Estados, regentando diversas temáticas. Si bien la OCDE tuvo buen recibo al comienzo de su gestión, está en mora de dar paso a países que, aunque no cumplen en su totalidad los preceptos del organismo, tienen el interés de hacerse participes de ellas, como es el caso de Colombia.

La OCDE, como la mayoría de organizaciones multilaterales, contiene un conjunto de elementos de toda naturaleza que deben ser pasados por la evaluación, seguimiento y monitoreo de los países que deciden acceder a ellos. Por lo general, los cambios en materia legislativa, normativa y de regulación emergen por cuenta de dichos organismos, ya sea por la vía de acuerdos, declaratorias, pactos, regulaciones o sanciones, pero para la mayoría de los sectores relacionados con estas temáticas, pareciera que esos temas sólo revisten interés desde el orden estatal, como en el caso colombiano, en virtud del impacto jurídico de la OCDE.

Poder establecer los lineamientos jurídicos, identificar la trazabilidad normativa, el orden de regulación y capacidad sancionatoria por cuenta de los parágrafos expuestos por la OCDE implica reconocer la trascendencia de los vínculos internacionales, y más cuando estos terminan lineando no sólo el sistema legislativo colombiano sino la capacidad de respuesta por la vía de la política pública. Por ende, se considera más que pertinente la revisión de este tema, no sólo por el diálogo e interés dispuesto por el Estado colombiano para engrosar la OCDE, sino porque representa el estado de cosas, poco tratado en el país.

La adhesión de Colombia a la OCDE cuenta en un alto de grado de importancia, prueba de ello es la recomposición del aparato institucional en materia de creación de agencias estatales (Ley 1444, 2011), la legislación para la lucha contra la 
corrupción, etc. Sin embargo, mientras estos obedecen a un recambio lógico de las exigencias planteadas por la OCDE al Estado colombiano, se presenta el interés por hacer monitoreo estatal sobre lo que implica la transformación jurídica que el país requiere para no solo hacer parte de la OCDE sino de los requisitos que por vía sectorial se le exigen para contar como parte de ella en sus revisiones.

La estructuración del artículo obedece a la siguiente composición: primero, se hace un diagnóstico del impacto jurídico de la OCDE sobre la base de los 23 capítulos que mide la organización; segundo, se estudia la capacidad de respuesta del Estado colombiano frente a las modificaciones jurídicas planteadas por la OCDE, y tercero, se analiza la capacidad de respuesta del Estado colombiano frente a la incorporación de la legislación sobre los sectores del país considerados estratégicos para las buenas prácticas, según la OCDE. En tal sentido, el escenario del análisis económico del derecho y del derecho anglosajón, propio, de un derecho más versátil, son las apuestas de cambio en los 23 comités.

\section{Diagnóstico del impacto jurídico de la OCDE sobre la base de los 23 capítulos que mide la organización}

La proliferación de distintos organismos posteriores a la Segunda Guerra Mundial, determinarían la arquitectura del orden económico y político mundial. Por un lado, se encuentra Occidente, blindado por un manejo económico liberal de cara al crecimiento económico para el desarrollo, y por el otro está Oriente, afincado en una dirección más centrada en la dirección del Estado en busca de igual propósito. La Organización de las Naciones Unidas (ONU); el Banco Internacional de Reconstrucción y Fomento (BIRF: Banco Mundial); el Fondo Monetario Internacional (FMI); el Acuerdo General de Aranceles y Comercio (GATT) y la Organización para la Cooperación y el Desarrollo Económico (OCDE) constituirían la base del orden internacional. 
Creada en 1961 por las 20 naciones económicamente más ricas del mundo, como un mecanismo de cooperación para promover políticas que "mejoren el bienestar económico y social de las personas alrededor del mundo", la OCDE ha jugado un papel fundamental en la expansión de la economía mundial hacia la liberalización del comercio internacional por medio de la cooperación económica entre las principales economías del mundo occidental, pero conservando ciertos privilegios económicos para sus miembros, siendo ésta la razón del celo de la membrecía. Hoy la integran 34 países miembros, cuenta con su sede en París, 2.500 funcionarios, un presupuesto anual de 342 millones de dólares y produce más de 250 publicaciones al año sobre temas de políticas públicas en materia económica. (Observatorio de Cooperación Internacional - Colombia, 2011, p. 1).

El origen de la OCDE parte del orden internacional establecido por los países desarrollados de la órbita occidental. Su propósito, vincularse a un organismo que, además de rector, aplicará en iguales términos una serie de ajustes y prebendas para viabilizar al Estado, pero, igualmente, las condiciones de la economía a través del mercado. En esencia, vendría a hacer la meta lograda por cuenta de los países luego del plan Marshall (Europa) o el plan MacArthur (Oriente), y que terminaría por enfilar las políticas estatales y empresariales para encaminar a una senda de crecimiento y prosperidad continua, cuyos frutos serían para la población perteneciente a ellos.

La OCDE es la continuadora de la Organización para la Cooperación Económica Europea (OEEC, por su sigla en inglés) creada en 1947 para administrar los fondos del Plan Marshall para la reconstrucción de Europa después de la Segunda Guerra Mundial. Sus miembros originales fueron 17 países de Europa Occidental, más Canadá, los Estados Unidos y Turquía, quienes suscribieron la Convención de la OCDE en 1960. Al igual que la OEEC, la OCDE tiene su sede en París. La OCDE es un foro en el cual los gobiernos de los países miembros «comparan experiencias de políticas públicas, buscan respuestas a problemas comunes, identifican buenas prácticas y coordinan políticas internas e internacionales». (Sáez, 2010, p. 94). 
En su momento inicial, conforme a su trayectoria, fue considerado un club selecto, sólo para los países que habrían podido resolver su dependencia económica de productos primarios, consolidándose en un principio de la mano de una importante base industrial que, posteriormente, los terminaría llevando a una posición de privilegio en materia de ciencia, tecnología e innovación. De ellas, la calidad y condiciones de vida de sus habitantes, a la par con las condiciones laborales, productivas y de consumo, vendrían a ser el fruto del balance positivo obtenido en los frentes de trabajo inicial. Aun así, seguía siendo el ejemplo a seguir para los países en vías de desarrollo.

La OCDE está, asimismo, a la vanguardia de los esfuerzos por entender y apoyar a los gobiernos a responder a los nuevos acontecimientos y temas, tales como la gobernanza corporativa, la economía de la información y los retos provocados por el envejecimiento de la población. La Organización ofrece un foro donde los gobiernos pueden compartir experiencias de políticas públicas, buscar respuestas a problemas comunes, identificar buenas prácticas y trabajar en la coordinación de políticas nacionales e internacionales. (García, 2010, p. 2).

La OCDE, entre sus diversos encargos para los Estados miembros, contiene la definición concreta de un marco regulatorio en la relación Estado-empresa con el que puedan postularse políticas públicas en la materia, entre otros ámbitos y sectores. $\mathrm{Al}$ conocerse el proceso de adhesión del Estado colombiano para ingresar a dicha organización, más allá de los señalamientos abocados al respecto, debe atenderse específicamente lo que implica reportar resultados en los temarios que hacen parte de los requisitos exigidos por la OCDE a sus miembros como base para aprovechar y sostenerse en el mencionado Club.

La OCDE ha establecido programas de "compromiso ampliado" con las economías emergentes más importantes, como son: Brasil, China, India, Indonesia y Sudáfrica, cuyo objetivo es su posible adhesión. En este sentido, los estudios e investigaciones sobre estos países no miembros ya no son una tarea marginal del Centro de Desarrollo Económico (CDE), sino que se han constituido en un tema central para el que se han establecido programas regionales en varios países de África, Asia y América Latina. (Clifton y Díaz, 2011, p. 1). 
La ruta emprendida para el proceso equivalía a entender que los países pasarían por las etapas de atraso, progreso y en vías de desarrollo. No obstante, las reconversiones establecidas para el logro de dichos preceptos terminaron siendo solo reciprocas para países cuya consistencia económica, política y social les permitió pasar por dichos escenarios, terminando, entre otros llamados, como países industrializados o post-industrializados, en los que se encuentran los pertenecientes a la OCDE. Los países que no alcanzaron esta senda de crecimiento y desarrollo tendrían que sobrellevar esta situación, en la que se entendería que unos países tendrían más éxito que otros.

Aunque agrupa a las principales economías del mundo, la OCDE es uno de los organismos internacionales menos conocidos y estudiados. Es fácil identificarla por la amplitud y variedad de los informes y estadísticas que publica, pero no es tan sencillo definir su estatus internacional ni la vinculación real que mantiene con los países que conforman la Organización. Se trata, fundamentalmente, de un organismo de cooperación internacional del que surgen normas no vinculantes, si bien su capacidad de influencia sobre los Estados miembros es más singular y compleja de lo que podría parecer a primera vista. En gran medida, esa complejidad tiene su origen en la propia forma de actuación de la OCDE; una forma de actuación institucional que reposa sobre tres elementos interrelacionados: a) la existencia de una configuración multilateral que estimula la formulación de propuestas y recomendaciones; $b$ ) la aplicación de una metodología de evaluaciones externas (inter-pares o peerreview) que permite valorar prácticas y comportamientos; c) la búsqueda de consensos que sirven de base para legitimar la influencia ejercida sobre los Estados miembros y minimizar los posibles conflictos (si bien este aspecto se ha visto facilitado en los últimos años, a medida que la OCDE se ha inclinado hacia el neoliberalismo y los países anglosajones han ganado peso en la Organización). (Nieto, 2011, pp. 41-42).

El precepto perseguido por la OCDE sintetiza el esfuerzo por ubicar y unificar la acción estatal con el interés de las empresas privadas y otros sectores para obtener mejores réditos de manera conjunta que por cuenta propia a través del logro y pre- 
disposición para la elaboración de políticas públicas conjuntas en diversos temas: medio ambiente, política económica e industrial, oportunidades a la población, formalización del empleo, cero corrupción, transparencia contractual, seguridad jurídica, entre otros, hacen parte de los criterios que persigue la OCDE para permitir el ingreso de países como miembros activos en ella ${ }^{2}$. La expectativa mediática está puesta en el Estado, pero ello involucra a las empresas.

Desde la Alta Consejería para la Gestión Pública y Privada se ha coordinado el proceso de acceso de Colombia a la OCDE, el club de buenas prácticas. Se creó un equipo conformado por los Ministros de Hacienda, Comercio, Justicia, Trabajo, TIC y Agricultura, entre otros. Así, se identificaron las prioridades para trabajar, de la mano con la OCDE, en temas de impuestos, empleo, medio ambiente, ciencia y tecnología, inversión y competencia, entre otros. Después de dos años de trabajo técnico y político, el 30 de mayo del año 2013 Colombia fue invitada a un proceso formal de acceso a la OCDE. El siguiente paso será la implementación de la Hoja de Ruta que nos ha dado la Organización, donde están listadas 23 áreas de política pública. Cada área es representada por un comité de expertos de la OCDE que tendrá que dar un visto bueno sobre Colombia. (Presidencia de la República, 2013, p. 9).

La OCDE se encuentra catalogada como un organismo multilateral capaz de transmitir, por la vía de políticas públicas, la carga legal que deriva de los comités que le hacen parte. En este sentido, lo que se recibe por lo general de la Organización es una carga jurídica o legal que debe considerarse para cada uno de los capítulos, comités o sectores. Por lo tanto, es claro que lo pretendido con la OCDE es mejorar las condiciones jurídicas de los países inscritos en ella, y para ello se requiere la recomposición de la plataforma jurídica recreada por el sistema jurídico OCDE.

\footnotetext{
${ }^{2}$ Decreto número 1400 (2011). Por el cual se establece el Punto Nacional de Contacto de Colombia y se adopta el procedimiento previsto en las Líneas Directrices de la Organización para la Cooperación y el Desarrollo Económico ("OCDE”) para Empresas Multinacionales. En: Pradilla (2012, p. 12).
} 


\begin{tabular}{|c|c|}
\hline Comité de Política Económica & Comité de Competencia \\
\hline $\begin{array}{l}\text { Comité de Revisión } \\
\text { Económica y Desarrollo }\end{array}$ & $\begin{array}{l}\text { Comité de Política } \\
\text { Científica y Tecnológica }\end{array}$ \\
\hline $\begin{array}{l}\text { Comité de Polìtica } \\
\text { Ambiental }\end{array}$ & $\begin{array}{c}\text { Comité de Políticas de } \\
\text { Información, Computación y } \\
\text { Comunicaciones }\end{array}$ \\
\hline $\begin{array}{c}\text { Comité de Sustancias } \\
\text { Químicas }\end{array}$ & $\begin{array}{c}\text { Comité de Política } \\
\text { sobre los Consumidores }\end{array}$ \\
\hline $\begin{array}{l}\text { Comité de Asistencia } \\
\text { al Desarrollo }\end{array}$ & $\begin{array}{l}\text { Comité de Industria y } \\
\text { Ambiente de Negocios }\end{array}$ \\
\hline Comité de Gestión Pública & Comité de Transporte Marítimo \\
\hline Comité de Comercio & Comité del Acero \\
\hline Comité de Inversiones & Comité del Turismo \\
\hline $\begin{array}{l}\text { Internacionales y Empresas } \\
\text { Multinacionales }\end{array}$ & $\begin{array}{c}\text { Comité de Empleo, Mano de Obra } \\
\text { y Asuntos Sociales }\end{array}$ \\
\hline $\begin{array}{c}\text { Comité de Movimientos de Capital } \\
\text { y Transacciones Invisibles }\end{array}$ & Comité de Educación \\
\hline Comité de Seguros & Comité de Agricultura \\
\hline Comité de Mercados Financieros & Comité de Pesca \\
\hline $\begin{array}{c}\text { Comité de Asuntos } \\
\text { Fiscales }\end{array}$ & $\begin{array}{c}\text { Comité para el Desarrollo de una } \\
\text { Politica TerritorialCuadro }\end{array}$ \\
\hline
\end{tabular}

Figura 1. Cuadro 2 OCDE: Los Comités Principales Fuente: De la Torre (2004, p. 63).

La OCDE emergió con la idea de postular los aspectos, criterios y elementos que, bajo condiciones de mercado, deberían alistarse para poder encausar de nuevo a los países hacia la senda del crecimiento y el desarrollo. En principio, la atomización adquirida en esta Organización sirvió para que se entendiera como un terreno solo allanado por países virtuosos en condiciones inmejorables a la hora de revisar las bases en que se apoyaron para el logro de tales escenarios. Sin embargo, las exigencias que les caracterizaban hacían casi que imposible poder considerar alguna opción de ingreso; por ende, se entendía que, además, era una organización de países ricos. 


\begin{tabular}{|c|c|c|}
\hline \multicolumn{3}{|c|}{ Organigrama de la OCDE } \\
\hline $\begin{array}{c}\text { Departamento } \\
\text { Económico }\end{array}$ & $\begin{array}{l}\text { Directorio de } \\
\text { Estadísticas }\end{array}$ & $\begin{array}{l}\text { Directorio para el } \\
\text { Medio Ambiente }\end{array}$ \\
\hline $\begin{array}{c}\text { Directorio de } \\
\text { Cooperación para el } \\
\text { Desarrollo }\end{array}$ & $\begin{array}{c}\text { Directorio } \\
\text { DesarrolloDirectorio de } \\
\text { Gobernabilidad Pública } \\
\text { y Desarrollo Territorial }\end{array}$ & $\begin{array}{l}\text { Directorio de } \\
\text { Comercio }\end{array}$ \\
\hline $\begin{array}{c}\text { Directorio para los } \\
\text { Asuntos Financieros } \\
\text { y de Empresas }\end{array}$ & $\begin{array}{c}\text { Centro para Políticas } \\
\text { Impositivas y } \\
\text { Administración }\end{array}$ & $\begin{array}{c}\text { Directorio para la } \\
\text { Ciencia, la Tecnología } \\
\text { y la Industria } \\
\end{array}$ \\
\hline $\begin{array}{c}\text { Directorio para el } \\
\text { Empleo, el Trabajo y } \\
\text { los Asuntos Sociales }\end{array}$ & $\begin{array}{l}\text { Directorio para } \\
\text { la Educación }\end{array}$ & $\begin{array}{c}\text { Directorio para la } \\
\text { Alimentación, la } \\
\text { Agricultura y la Pesca }\end{array}$ \\
\hline $\begin{array}{l}\text { Directorio de } \\
\text { Asuntos Públicos y } \\
\text { Comunicaciones }\end{array}$ & Directorio Ejecutivo & $\begin{array}{l}\text { Centro para la } \\
\text { Cooperación con } \\
\text { No Miembros }\end{array}$ \\
\hline \multicolumn{3}{|c|}{ Otros Cuerpos } \\
\hline $\begin{array}{c}\text { Agecia Internacional } \\
\text { de Energía }\end{array}$ & $\begin{array}{c}\text { Agencia de Energía } \\
\text { Nuclear }\end{array}$ & $\begin{array}{c}\text { Conferencia Europea } \\
\text { de Ministros de } \\
\text { Transporte }\end{array}$ \\
\hline $\begin{array}{l}\text { Centro para el } \\
\text { Desarrollo }\end{array}$ & $\begin{array}{c}\text { Centro para la } \\
\text { Investigación e } \\
\text { Innovación Educativa }\end{array}$ & $\begin{array}{c}\text { Club del } \\
\text { Sahel y África } \\
\text { OccidentalCuadro }\end{array}$ \\
\hline
\end{tabular}

Figura 2. Estrcuctura OCDE

Fuente: De la Torre (2004, p. 62).

El "Club de buenas prácticas", como de manera iterativa se llama a la Organización, implica para los integrantes de la misma la imperiosa necesidad de actuar desde los mayores estándares ambientales, económicos, legales, políticos y sociales. Se trata de una manera de elevar la capacidad de la gestión estatal para que los actores individuales y privados puedan igualmente incrementar sus respectivas condiciones y estándares. En esencia, se pretende, en la OCDE, elevar el papel que cumplen el Estado, las empresas y los particulares para impulsar mejores oportunidades colectivas, individuales y sociales de cara al impacto de las políticas públicas que entre ellos se dispongan por la vía de la incorporación del patrimonio legal OCDE. 
El concepto de acquis de la OCDE es relevante para entender lo que deben hacer los países que postulan a ser miembros. La OCDE ha definido el acquis como el conjunto de instrumentos legales de la Organización, que no solo incluyen las decisiones y recomendaciones aprobadas por el Consejo sino también instrumentos internos, como las resoluciones del Consejo, y externos, como las declaraciones ministeriales, entre otras. La palabra francesa acquis podría traducirse entonces como «patrimonio legal». Todo país candidato a ser miembro debe suscribir o aceptar este patrimonio legal. La forma en que puede hacerlo depende del instrumento respectivo. (Sáez, 2010, p. 98).

Es evidente que el gran protagonismo en la materia lo tienen los Estados y las empresas, desde las que efectivamente pueden alcanzarse los postulados que atienden las razones por las cuales ha sido creada la OCDE. La excelente interacción en ambas frentes de trabajo puede traer mejores réditos para la población en materia laboral, productiva y social, propósito que requiere la definición de unos acuerdos, lineamientos y pactos para que las acciones cometidas en un sector tengan ascendencia en los otros de manera recíproca y simultánea. Así que, al ubicar a los dos primeros agentes bajo esta responsabilidad, se extiende la posibilidad de alcanzar los parámetros exigidos.

La OCDE ayuda a los gobiernos a promover la prosperidad y a combatir la pobreza a través del crecimiento económico, la estabilidad financiera, el comercio y la inversión, la tecnología, la innovación, el estímulo empresarial y la cooperación para el desarrollo. Procura que se tomen en cuenta las implicaciones ambientales del desarrollo social y económico. Otros objetivos incluyen la creación de empleos para todos y la equidad social, así como lograr una gobernanza transparente y efectiva. (OCDE, 2007, p. 7).

La OCDE fomenta la gestión pública-privada de manera unívoca para lograr los resultados en materia de políticas públicas acompasadas por la participación de dichos frentes estamentales e institucionales. Al respecto, es importante que en Colombia se atienda claramente lo que implica el hecho de 
pertenecer a un club de países para los que las propuestas y resultados tienen corresponsabilidad en los sectores más representativos de cualquier país. Estado y empresa, para el caso colombiano, terminan siendo evaluados de la misma manera y no como agentes con intereses particulares; por ende, la relevancia de establecer el fundamento que se requiere para pertenecer a la misma y de paso contribuir a ella en otros campos.

A la hora de revisar la técnica jurídica empleada por el legislador para implementar la OCDE, llama la atención que no se haya considerado un tipo penal inverso, es decir, un sistema de responsabilidad penal de las personas naturales (director, socio, presidente, administrador) en el evento que la persona jurídica haya cometido uno de los delitos comentados y su administración haya consentido o haya tolerado la conducta ilícita. (Merino, 2002, pp. 328-329).

El interés de la OCDE para que en los países miembros las "mejores prácticas" se traduzcan en un mayor bienestar y competitividad para sus habitantes pasa por la retina del buen gobierno (gobernanza), la corrupción, la transparencia entre otros campos, que implican la seriedad con que se realizan diversas actividades en los estados miembros, en los que efectivamente, además de funcionar y operar, los distintos estamentos e instituciones obtienen resultados contundentes que impulsan a toda la sociedad. En ellas, se pretende ubicar a cada país en la posibilidad de lograr el anhelado desarrollo o posdesarrollo y/o mejorar prácticas.

La tarea que corre por cuenta del Estado colombiano y de los sectores involucrados consiste en dar a conocer de manera vertiginosa lo que implica el hecho de pertenecer a la OCDE. No puede obviarse la animadversión que se tiene en el país en cuanto a los marcos de política internacional o prerrogativas provenientes de los diferentes organismos multilaterales acusados de profundizar, aún a través de sus recetas, las dificultades por las cuales están atravesando los países que no han podido resolver, entre otros aspectos; un modelo económico con el cual puedan desarrollarse efectivamente. . 
Tabla 1

TEMAS DE INTERÉS DE LA OCDE.

\begin{tabular}{|c|c|}
\hline \multirow{6}{*}{ ECONOMÍA } & Agricultura \\
\hline & Comercio \\
\hline & Competencia \\
\hline & Desarrollo Regional, Rural y Urbano \\
\hline & Economía y Crecimiento \\
\hline & Empresa, Industria y Servicios \\
\hline DESARROLLO & Temas relacionados con el Desarrollo \\
\hline \multirow{4}{*}{ GOBERNANZA } & Reforma Regulatoria \\
\hline & Gobernanza Pública \\
\hline & Gobiernos Corporativos \\
\hline & Lucha contra la Corrupción \\
\hline \multirow{4}{*}{ SUSTENTABILIDAD } & Desarrollo Sustentable \\
\hline & Energía \\
\hline & Medio Ambiente \\
\hline & Pesquerías \\
\hline \multirow{5}{*}{ SOCIEDAD } & Educación \\
\hline & Empleo \\
\hline & Migraciones \\
\hline & Salud \\
\hline & Temas Sociales y de Bienestar \\
\hline \multirow{4}{*}{ FINANZAS } & Inversión \\
\hline & Mercados Financieros \\
\hline & Seguros y Pensiones \\
\hline & Tributación \\
\hline \multirow{3}{*}{ INNOVACIÓN } & Biotecnología \\
\hline & Ciencia e Innovación \\
\hline & Tecnologías de la Información y de las Comunicaciones \\
\hline
\end{tabular}

Fuente: Sáez (2010, p. 110). 
La capacidad de respuesta del Estado colombiano frente a las modificaciones jurídicas planteadas por la OCDE

El salto de país fallido e inviable hacia la posibilidad de integrar el selecto grupo de países OCDE es una apuesta concreta al interés de transformar las instituciones en Colombia. En esencia, se busca que las condiciones e interacciones en las relaciones Estado, empresa y sociedad estén reguladas por los mayores estándares de idoneidad, resultados y transparencia en los aspectos que atañen a cada uno de ellos en la relación. La OCDE ubica a las políticas públicas como el emblema que caracteriza dicha interacción, y que, al paso de ellas, pone en evidencia la reducción de las desigualdades e inequidades, precisamente, con el trabajo mancomunado de dichos agentes.

La Alta Consejería para la Gestión Pública y Privada, en desarrollo de las funciones establecidas en el Decreto 3445 de 2010, ha enfocado sus esfuerzos en desarrollar diferentes estrategias tales como: la coordinación del Sistema Nacional de Competitividad, la coordinación de la Mesa Transversal de Empleo, el proceso de ingreso de Colombia a la OCDE, la coordinación de la Política Anti trámites, la coordinación de la Adquisición e Implementación del Sistema de Inspección No Intrusiva de Mercancías (Escáneres), y la facilitación del desarrollo de Buenaventura como nodo logístico del Pacífico. (Presidencia de la República, 2013, p. 7).

La cuestión radica en identificar los puntos que para el Estado y las empresas en Colombia o los principales sectores económicos del país podrían influir en la consideración de un panorama de trabajo en materia de políticas públicas. De lograrse ello, problemáticas como la captura pública, la selección adversa y el riesgo moral y los costos sociales de transacción serían superados. De darse, en Colombia se podría hablar de nuevas y mejores prácticas no solo en la relación Estado-empresa sino también incluyendo al ciudadano para materializar efectivamente la participación de la población. 
Tabla 2

LA ECONOMÍA DE COLOMBIA EN PERSPECTIVA.

\begin{tabular}{|c|c|c|c|c|c|c|c|c|c|c|}
\hline \multirow{2}{*}{ Categoría/Indicador } & \multirow{2}{*}{ Unidades de Medida } & \multirow{2}{*}{ Colombia } & \multirow{2}{*}{$\begin{array}{c}\text { Promedio de países LAC } \\
\text { (América Latina y el Caribe) }\end{array}$} & \multicolumn{4}{|c|}{ Países de la OCDE } & \multicolumn{3}{|c|}{ Puesto de Colombia } \\
\hline & & & & Mìnimo & Media & Mediano & Máximo & Mundo & OCDE & LAC \\
\hline \multicolumn{11}{|l|}{ Tamaño del país } \\
\hline Superficie & $100 \mathrm{~km}^{2}$ & 1142 & 601 & 3 & 1063 & 188 & 9985 & 26 & 5 & 5 \\
\hline Población & 1000 & 46927 & 17493 & 319 & 36623 & 10823 & 311592 & 28 & 10 & 3 \\
\hline Mano de obra & 1000 & 22136 & 9353 & 188 & 17748 & 5280 & 157493 & 30 & 11 & 3 \\
\hline \multicolumn{11}{|l|}{ PIB } \\
\hline A la tasa de & $\begin{array}{c}\text { mil } \\
\text { millones }\end{array}$ & & & & & & & & & \\
\hline cambio actual & $\begin{array}{l}\text { USD } \\
\text { mil }\end{array}$ & 332 & 172 & 14 & 1356 & 499 & 15094 & 32 & 21 & 4 \\
\hline $\begin{array}{c}\text { En PPP, } \\
\text { USD actuales }\end{array}$ & $\begin{array}{l}\text { millones } \\
\text { USD }\end{array}$ & 474 & 225 & 11 & 1281 & 366 & 15094 & 26 & 15 & 4 \\
\hline Comercio & $\begin{array}{c}\text { mil } \\
\text { millones }\end{array}$ & & & & & & & & & \\
\hline exterior & USD & 124 & 74 & 15 & 790 & 449 & 4770 & 51 & 31 & 6 \\
\hline \multicolumn{11}{|l|}{$\begin{array}{l}\text { Indicadores } \\
\text { de desarrollo }\end{array}$} \\
\hline PIB per cápita & USD & 7067 & 8601 & 10064 & 40387 & 40598 & 115039 & 88 & 35 & 18 \\
\hline $\begin{array}{c}\text { A la tasa de } \\
\text { cambio actual }\end{array}$ & USD & 10103 & 11196 & 15340 & 34973 & 34736 & 88787 & 85 & 35 & 17 \\
\hline $\begin{array}{c}\text { Índice de } \\
\text { Desarrollo Humano }\end{array}$ & & 0,710 & 0,731 & 0,699 & 0,871 & 0,943 & 0,943 & 87 & 34 & 22 \\
\hline
\end{tabular}

Nota: En la fila de comercio exterior se indica la suma de las exportaciones y las importaciones, en USD.

El índice de desarrollo humano es un índice medido en una escala de $0=$ más bajo a $1=$ valor positivo más alto.

ALC (América Latina y el Caribe), según el Banco Mundial, exceptuando a siete paises para los que no se

dispone de datos recientes (Aruba, Islas Caimán, Curazao, San Martín, Islas Turcas y Caicos, Islas Vírgenes).

Fuente: Banco Mundial (2013) 
Es el momento para que en Colombia sea medida la capacidad estamental e institucional que tienen los frentes estatales y empresariales para contribuir a elevar las condiciones económicas, legales, políticas y sociales del país en la medida que atiendan los requisitos que al respecto exige la OCDE. Es la primera vez que el país pone a prueba el orden institucional, en tanto, el Estado, las empresas y la sociedad misma, para encauzar las disposiciones de política estatal, política empresarial y política social para poder superar las dificultades que han impedido que el país logre desarrollarse para todos.

\begin{abstract}
Ahora, desde la orilla de los analistas, casi todos coinciden en que el país no tiene hoy los pergaminos en sus indicadores sociales, económicos, ambientales y demás, como para ser ya un miembro de la OCDE. No obstante, los altos estándares por cumplir para estar a la altura de la OCDE, ven con buenos ojos que la adopción de sus recomendaciones sea la forma en que Colombia gane en cuatro aspectos. En primer lugar, una mayor visión de futuro que amplía el horizonte de llegar a ser un país desarrollado. También se asegura la continuidad de las políticas públicas de un gobierno a otro, al tiempo que se mejora la calidad de las políticas públicas. Lo tercero toca el crecimiento económico al enviar señales internacionales para que haya mayor confianza inversionista. Por último, las buenas nuevas de estar en la OCDE, solo se traducirán en beneficios tangibles en la calidad de vida del ciudadano de a pie más a largo que a corto plazo. (Rojas, 2013, p. 18).
\end{abstract}

Se ha convertido cotidiano durante los últimos años, hasta la vigencia del 2017, que Colombia viene dando pasos para ingresar al "Club de las mejores prácticas" OCDE. A medida que se avanza en cada uno de los capítulos evaluados por dicho organismo, sale a la luz pública el avance, contenido o resultado obtenido por el país en la materia. No obstante, no se conoce en general las implicaciones que tendría para el país en materia jurídica la entrada a la OCDE, y lo que ello podría acarrear para diversos sectores de la realidad nacional, por ende, el interés de tomar como referencia en materia legislativa a la OCDE y su trascendencia en el país. 


\section{Áreas de Trabajo}

\begin{tabular}{|c|c|c|}
\hline$凶$ Agricultura & $\otimes$ Desarrollo Sustentabley Crecimiento & $\otimes$ Gobierno Corporativo \\
\hline \Asuntos Sociales de Bienestar & Verde & $\triangle$ Industria Pesquera \\
\hline Q Biotecnologiay Seguridad Química & ه Economía y Crecimiento & QInversiones \\
\hline$\bigotimes$ Ciencia y Tecnologia & 凶Educación & $\square$ Medio Ambiente \\
\hline$凶$ Combate a la Corrupción & QEmpleo & Q Mercados Financieros \\
\hline 囚 Competencia & QEmpresa, Industria y Servicios & 凶Migración \\
\hline$\otimes$ Comercio & \Energía & ¿Salud \\
\hline QDesarrollo & $\triangle$ Fiscal & $\bowtie$ Segurosy Pensiones \\
\hline$\otimes$ Desarrollo Regional, Ruraly Urbano & \Gobiemo y Administración Pública & $\begin{array}{l}\text { Q Tecnologia de la Información y de las } \\
\text { Comunicaciones }\end{array}$ \\
\hline
\end{tabular}

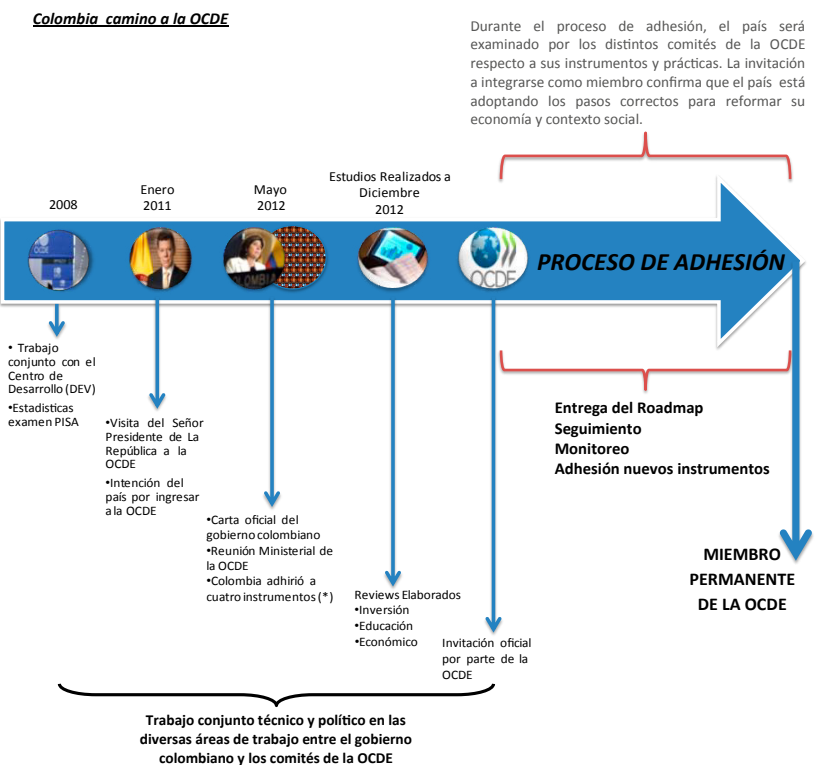

Figura 3. Colombia, camino a la OCDE.

Fuente: Embajada de Colombia en Francia (s.f).

La idea es precisamente poder ilustrar lo que implica no sólo para el Estado colombiano, sino para las entidades territoriales, como los departamentos y municipios, el hecho de asumir las unidades de medida y ponderación planteadas por los comités de la OCDE. En este sentido, se trata igualmente de dar preeminencia a los elementos que, desde la OCDE, terminan siendo transferidos por el Estado colombiano a los entes territoriales y la capacidad de estos para dar respuesta a dicha convocatoria y acuerdo internacional. No sólo se trata de réplicas entre dos estamentos internacionales sino las implicaciones que traen en materia para las entidades que subyacen a estas, como para el caso colombiano. 


\section{Desde la Presidencia de la República (2015), se considera- ron los siguientes aspectos:}

¿Cuáles son los requisitos para ingresar a la OCDE?

Para que Colombia ingrese a la OCDE debe demostrar que cumple (o está en el proceso de cumplir) los principios y estándares de la Organización que son comunes a los países miembros. Esto implica que el país es evaluado en los distintos Comités que la OCDE ha identificado como críticos para el país (23 Comités).

Los requisitos de entrada dependen del avance del país en los temas que aborda cada Comité y en los temas que el Comité ha identificado y anunciado como obligatorios. En este aspecto es clave diferenciar entre las recomendaciones que hace la OCDE en sus documentos de Revisión entre pares y lo que efectivamente ha anunciado cada Comité como requisitos de entrada. Esto implica que no todas las recomendaciones son obligatorias sino, en muchos casos, son aspectos deseables o buenas prácticas que el país puede considerar.

\section{¿Cuáles son los pasos para ingresar a la OCDE?}

El proceso de ingreso a la OCDE comienza con la voluntad del país para ser parte de la Organización.

Una vez se hace explícita esta solicitud, la OCDE inicia el acercamiento con el país solicitante y trabaja conjuntamente con el mismo en la generación de reportes y evaluaciones preliminares de sus políticas públicas. El Consejo de la Organización toma la decisión (por consenso) de invitar al país a iniciar el proceso formal de acceso.

La OCDE define la hoja de ruta y los estándares bajo los cuales el país será evaluado. Esta hoja de ruta es entregada al país.

El país debe presentar un Memorando Inicial en el cual expone el estado actual de sus políticas vs. los estándares de la Organización.

El Secretariado de la OCDE inicia un proceso de evaluación de las políticas públicas, a través de cuestionarios y/o misiones al país.

Las evaluaciones son presentadas ante los Comités, que reúnen a todos los delegados de los países miembros.

El país debe presentar y sustentar avances ante los Comités, durante las rondas que resulten necesarias, para lograr su aprobación.

Una vez todos los Comités den su aprobación, el Consejo de la OCDE será el encargado de dar la decisión final para el acceso del país a la Organización.

Una vez se dé la aprobación por parte de la OCDE, será el Congreso de la República quien ratifique y apruebe su ingreso, con revisión automática de la Corte Constitucional, como cualquier tratado internacional.

\section{Cuadro Resumen 1. Requisitos para ingresar a la OCDE.}

Fuente: Presidencia de la República, (2015). 


\section{Asimismo Gutiérrez (2014), expresa;}

Colombia tiene el reto de entrar a la Organización para la Cooperación y el Desarrollo Económicos (OCDE) en un plazo de dos años, pero para lograrlo debe trabajar en 23 tareas que ese organismo ha planteado. En la OCDE hay más de 200 comités de diversos temas, pero Colombia debe darles prioridad a 23 de ellos.

Catalina Crane dice que el ingreso a ese club podría darse en un plazo de 24 meses, pero advierte que todo dependerá de las evaluaciones que haga el organismo internacional sobre Colombia. "Todo este proceso de evaluaciones toma su tiempo. Si todo fluye muy bien y se pueden hacer todas las evaluaciones, y no hay que hacer otras, se podría hacer todo en un plazo de dos años. Sería posible si todo funcionara perfecto. Algunos países se han tomado tres años", dice Crane. Recuerda que en enero del 2011 el presidente Santos anunció el interés de entrar a la organización, y el país fue invitado formalmente en mayo del 2013. Ahí se inició el proceso.

\section{Tala Regulatoria}

Un aspecto que la OCDE considera clave es la estructura regulatoria de Colombia, la cual debe ajustarse para hacerla más coordinada."Tenemos buenas entidades regulatorias, pero trabajan aisladas: transporte, medioambiente, petróleo, servicios públicos, etc.; pero nos han dicho que todas se deben articular", señala Crane.

La OCDE termina por orientar el accionar del Estado colombiano en materia jurídica, económica, política, social y de relaciones internacionales desde la perspectiva de metas, políticas públicas y valores en los que cabe perfectamente el interés de cumplir los propósitos de la relación Estado-población por encima de los limitantes de la legislación, llevando a estas la posibilidad de orientar el impacto, resultados y trascendencia del Estado por la vía de la ejecución de proyectos de política pública y, en general, tratando de que se materialice la idea de que el Estado debe dedicarse a sus funciones, dejándole el resto a los particulares o la responsabilidad a la sociedad. 
Capacidad de respuesta del Estado

colombiano frente a la incorporación

de la legislación sobre los sectores

conforme a la OCDE

Es importante para la reorientación jurídica, legislativa y regulatoria del país que se atiendan las referencias enmarcadas en la OCDE. No se trata solo de reglar conductas, normas o principios, se trata de poner en evidencia qué tanta coherencia se tiene en materia de legislación y los sectores sobre los cuales estos terminan insertos, a su vez, qué grado de reciprocidad entre dicho fenómeno se presenta, y de ser el caso, dar espacio para que se presenten condiciones de regulación entre lo legislativo y el sector a tutelar. Por ende, la cuestión se cierne en revisar las capacidades que se tienen en el país para acoger un acuerdo que, francamente, da un giro legislativo completo.

Tabla 3

Principales conclusiones y recomendaciones de la OCDE para Colombia.

\begin{tabular}{|c|c|}
\hline $\begin{array}{c}\text { PRINCIPALES } \\
\text { CONCLUSIONES }\end{array}$ & $\begin{array}{c}\text { PRINCIPALES } \\
\text { RECOMENDACIONES }\end{array}$ \\
\hline \multicolumn{2}{|c|}{ Seguir fortaleciendo la resiliencia macroeconómica } \\
\hline $\begin{array}{l}\text { El sistema financiero } \\
\text { es sólido pero algunas } \\
\text { vulnerabilidades crean } \\
\text { riesgos. }\end{array}$ & $\begin{array}{l}\text { Aprobar la ley que dota a la } \\
\text { Superintendencia Financiera } \\
\text { de poderes regulatorios } \\
\text { sobre sociedades holding de } \\
\text { conglomerados financieros. }\end{array}$ \\
\hline $\begin{array}{l}\text { Es probable que los gastos } \\
\text { necesarios en infraestructura } \\
\text { y previsión social excedan } \\
\text { a los previstos en el marco } \\
\text { fiscal para el mediano plazo. }\end{array}$ & $\begin{array}{l}\text { Recaudar más ingreso en el } \\
\text { mediano plazo. }\end{array}$ \\
\hline
\end{tabular}


Mantener un fuerte crecimiento económico

Las deficiencias en la infraestructura suben los costos del transporte.

El desempeño en innovación es débil como resultado de la escasa inversión en I+D y la vinculación insuficiente entre el sector privado y el académico.

La competitividad de las empresas se ve obstaculizada por cargas regulatorias y resultados judiciales lentos.
Destinar más apoyo público a la formación de competencias y habilidades en regiones rezagadas. Establecer un programa nacional para la educación escolar y profesionalizar las carreras de maestro y profesor.

Mantener el aumento en la inversión pública. Financiar más programas de infraestructura a escala regional. Implementar el programa de concesiones viales (4G9 y garantizar que las asociaciones Público-Privadas continúen siendo sometidas a los análisis de costobeneficio adecuados.

Brindar más subsidios y préstamos para I+D a las empresas.

Financiar proyectos de I+D que acerquen a la industria y al sector académico.

Eliminar regulaciones sobre la propiedad pública y la integración vertical en el sector de la electricidad, la integración vertical y la estructura del mercado en el sector ferroviario.

Introducir un tribunal o una división del tribunal dedicados integramente a fallos comerciales y facilitar la gestión de casos a través de herramientas digitales de manejo de casos.

La poca participación en cadenas globales de valor limita la adquisición de tecnología de vanguardia.
Facilitar la información de fallos anticipados sobre condiciones de importación de manera más agil y con mayor visibilidad. 


\begin{tabular}{l} 
Hacia un crecim \\
\hline \\
El alto grado de informalidad \\
agraba las desigualdades en \\
la medida en que limita el \\
acceso a beneficios públicos.
\end{tabular}

Mujeres no cuentan con suficientes oportunidades en el mercado laboral formal o informal.

Resultados educativos dependen fuertemente del fondo socioeconómico familiar.

Continúa habiendo grandes diferencias entre los niveles de pobreza rural y urbanos.
Reducir aún más los impuestos y contribuciones sobre salarios (costes laborales no salariales). Simplificar los procedimientos de registro de empresas y la afiliación de trabajadores a la seguridad social.

Establecer un diálogo social para discutir una diferenciación del salario mínimo según la edad y la región.

Asegurar la prestación accesible de una protección social de buena calidad para niños y de cuidados de larga duración para los parientes mayores o personas con discapacidad.

Ampliar el acceso a programas de medidas activas del mercado laboral y aprovecharlos mejor.

Prestar mayor apoyo público para aumentar la tasa de escolarización de los niños más desfavorecidos en las regiones menos desarrolladas. Expandir la educación de la primera infancia.

Fuente: OCDE (2017, p. 7).

La indiferencia que ha tenido para los jurisconsultos la revisión de los tratados vinculantes o acuerdos planteados con organismos multilaterales para con Colombia muestra que no sólo se está ad portas de ingresar a un club de buenas prácticas, sino que el marco jurídico tiene por tendencia girar hacia la regulación jurídica, marco bajo el cual no tiene racero la formación jurídica en el país. En una primera medida, la legislación colombiana ha venido incorporando elementos del derecho anglosajón 
al continental, de paso, ha terminado por dar más espacio a la jurisprudencia que a la legislación para, finalmente, integrar acuerdos o pactos como la OCDE en cuanto a regulación.

En ese orden de ideas, se han efectuado estudios, investigaciones pertinentes, tal como lo expresa en el siguiente cuadro resumen la Universidad EAFIT (2016):

¿Por qué Colombia quiere entrar?

Según explica Carolina Vásquez, para reforzar su papel en cuanto a relaciones internacionales y política exterior, a través de una mirada hacia lo biodiverso y cambiante.

¿Cómo va la candidatura?

Desde 2008, Colombia inició su campaña para ingresar a la OCDE, sin embargo, fue hasta 2013 que la organización aceptó su candidatura. Para aceptar la candidatura de un país, la OCDE establece una hoja de ruta, la cual establece que de los 250 comités y grupos de trabajo que tiene la organización, 23 se encargarían de revisar las políticas públicas del candidato, esto con el fin de evaluar si sus estándares van por el camino correcto o se acomodan a los de la Organización.

Para el caso de Colombia, de los 23 comités asignados, 12 ya dieron su visto bueno lo que, según Pelau, indica que tiene un norte claro para alcanzar los estándares de la OCDE. Sin embargo, de los 11 restantes a los que se enfrenta Colombia hay algunos particularmente complicados: el comité de relaciones laborales, el comité de empresas públicas, el comité de comercio y el comité de ambiente.

Problemáticas colombianas diagnosticadas por la OCDE:

La informalidad laboral genera pobreza, desigualdad y baja productividad.

El sistema tributario tiene poco recaudo y no favorece a la inversión.

El sistema de pensiones cuenta con poca cobertura.

Falta de inversión en investigación y desarrollo.

Depende de las exportaciones de materias primas.

"En la década de 1970 el $80 \%$ del PIB mundial estaba en los países que pertenecían a la OCDE, en el 2016 el $49 \%$ del PIB mundial hace parte de los países que están en la OCDE”, Nicolás Pelau. 
Cont...

Colombia en la OCDE.

\section{Beneficios de ingreso para Colombia}

Estudios y análisis. La OCDE es una organización de una alta sofisticación técnica que realiza evaluaciones de fondo y procesos de consultoría muy profundos donde revisa todo lo sucede en el país.

Voz y voto en discusiones globales. Además de la posibilidad de intervenir en el diseño de estándares internacionales en temas referentes a políticas públicas que pueden ser replicados en los países miembros.

Atracción de inversión extranjera. Al ingresar a la OCDE los países adquieren un sello de calidad que valida sus fortalezas institucionales, esto incide en la decisión de inversionistas extranjeros.

Impulso en la agenda pública. Principalmente en temas de fortalecimiento institucional, ingresar a una organización permite apalancar en las agendas públicas temáticas relacionadas con reformas políticas.

\section{Riesgos de ingreso para Colombia}

Posibles crisis financieras. Esto teniendo en cuenta el nivel de desarrollo del país, en términos de productividad y competitividad, respecto a los demás miembros de la organización nivel similar de desarrollo, de productividad y de competitividad.

Austeridad. Además de la reducción del gasto público, podrían llevarse a cabo privatizaciones, despidos y disminución en los salarios de los empleados.

Costos. Ingresar a la OCDE le representa a Colombia cerca de cinco millones de euros anuales en implementar sus recomendaciones para acercarse a los niveles de la organización, cerca de 20 billones de pesos anuales durante los próximos 15 años. Este dinero debe salir de los impuestos, por lo que sería inminente una reforma tributaria.

\section{Cuadro Resumen 2. Colombia, ¿en el "club de los países ricos”?}

Fuente: Universidad EAFIT, (2016).

Por lo tanto, al considerar a la OCDE como marco de referencia para el análisis jurídico que sobrevienen para el país implica reconocer en ella la posibilidad que, en adelante, los temas jurídicos de naturaleza estatal que no impliquen conflic- 
tos internacionales serán tratados por la vía de las políticas públicas, sin descuidar, claro está, el orden jurídico. No obstante, estas últimas pasaran a ser custodias de las políticas públicas y no propiamente el orden direccional de las acciones del Estado. Por ello, la OCDE expone claramente que el Estado debe abandonar por completo su presencia en escenarios donde no tiene cabida.

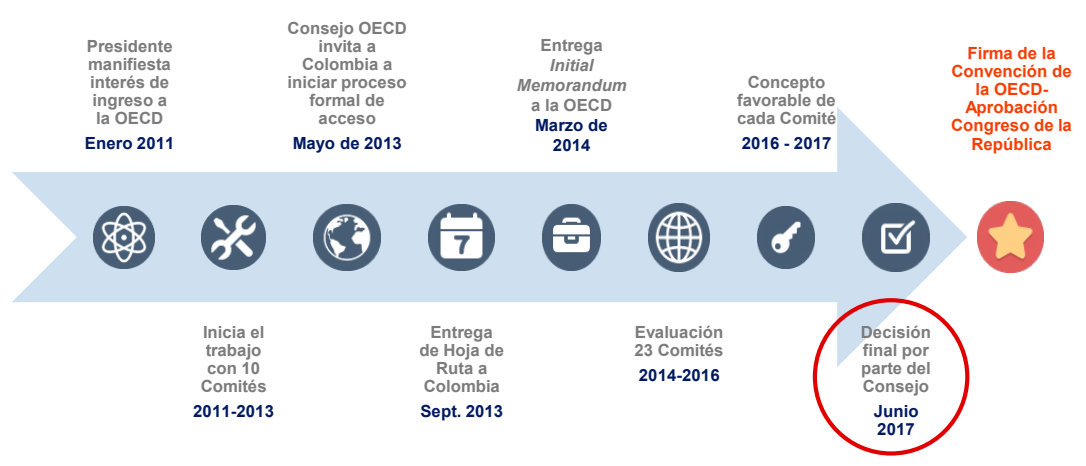

Figura 4. El camino de la Colombia a la OCDE.

Fuente: Presidencia de la República (2017, p. 8).

Es por ello que la formación jurídica se encuentra en ciernes a la hora de acoger la fundamentación de la OCDE, la cual traslada dicha incorporación de políticas públicas por la vía de su formulación. Por ende, puede decirse, sin lugar a dudas, que se está ante la posibilidad provechosa de dar un giro no solo a la manera cómo han sido atendidos los problemas en el país, sino sobre la manera cómo el juicio jurídico debe entrar a considerar dichos temas como tendencia de largo plazo.

Para poder ingresar a este 'club' se requiere un análisis riguroso de la legislación, las políticas y las prácticas del país respectivo en una gama muy amplia de temas (como medioambiente, inversión extranjera, gobernanza pública o agricultura); estos análisis se ocupan de hasta dónde esas normas o prácticas contribuyen a impulsar el crecimiento económico, a reducir las desigualdades, a mejorar la educación o a aumentar la transparencia y la eficiencia del Gobierno. (González-Pérez, 2017). 
La idea material de enfocar las prácticas estatales en el marco de la OCDE conlleva a que se sobreentienda que el país pasará de ser guiado, o recibir recursos, a cumplir, hacer parte de y de plantear prácticas que mejoren el quehacer estatal en sus diferentes divisiones centrales, regionales y locales. Por ello, se pasará del recibo de cooperación internacional en cualquier ámbito, a hacer recibo de reglas para mejorar todo lo que atañe la concepción de lo estatal. No obstante, es de aclarar que, si bien es una apuesta de orden estatal multilateral, el recibo de estas recaerá en los entes territoriales que igualmente deben prepararse para orientarse bajo los lineamientos de la relación OCDE - DNP.

\section{Conclusiones}

El país requiere revisar con profundidad lo que implica la adhesión a los 23 comités de la OCDE en vista del poco interés despertado por los sectores a los que de manera directa atiende o hace referencia el organismo multilateral. Frente a ello, sigue haciendo carrera en el país, la idea que refrenda el poco interés que despiertan estos temas dada la naturaleza multilateral, pero lo que en realidad encierra es una serie de ajustes que tienen implicaciones directas sobre la realidad sectorial del país, que en muchos de los casos muestra estar ajena o desatendida de lo que implica la implementación de dichos capítulos o comités en la estructura sectorial.

Es conveniente que se considere relevante acoger el análisis jurídico experimental sobre temas en los que el país o el Estado colombiano deben poner atención por el grado de trascendencia legal que tienen los acuerdos considerados. De paso, se pretende comparar qué tanto acople tiene la naturaleza de dichos comités en el ámbito nacional para, finalmente, poder vislumbrar qué tanta transformación jurídica o legislativa requiere el país para dar cuenta de dichos comités y, en consecuencia, establecer marcos de política pública para alinear estos a los intereses generales y de la OCDE. 
El desajuste presentado en la hoja de ruta que animaba el alcance simultáneo del desarrollo a través de recetas comunes aplicadas en los países terminó por irrumpir en la escena de los países. El éxito alcanzado por unos países no implica el acervo para otros o la perduración de una situación precaria. En algunos países se ha entendido que el desarrollo se logra de manera efectiva a medida que ellos, además de acometer sus tareas, colaboran, impulsan y promueven de la mano a otros países para revisar las condiciones que les impiden alcanzar mejores niveles y rubros en materia de desarrollo. Este es el caso de la OCDE, que aún expone el posible alcance de dicho contexto.

\section{REFERENCIAS}

Banco Mundial PNUD. (2013). Programa de las Naciones Unidas para el Desarrollo. Indicadores del desarrollo Mundial.ONU

Clifton, J. y Díaz, D. (2011). The New Political Economy of the OECD in a context of Shifting World Wealth. Munich Personal RePEc Archive, (33010). pp. 1-24. Recuperado de https://mpra.ub.uni-muenchen.de/33010/1/MPRA_paper_33010.pdf

De la Torre, E. (2004). La OCDE: un faro de occidente para un mundo en transición. Agenda Internacional (6). 54-87. Recuperado de https://www.agendainternacional.com/numerosAnteriores/n6/0604.pdf

Embajada de Colombia en Francia. (s.f.). Colombia, camino a la $O C D E$. [En línea]. Recuperado de http://wsp.presidencia. gov.co/ocde/documentos-realizados/Documents/Colombiacamino-ocde.pdf

García, J. P. (2010). Prácticas y políticas exitosas para promover la mejora regulatoria y el emprendimiento a nivel subnacional. Documentos de trabajo de la OCDE sobre gobernanza pública, 2010/18. OCDE. 
González-Pérez, M. A. (2017, 8 de octubre). Así va el proceso de Colombia para ingresar a la OCDE. Un balance sobre la situación en la que está el país para entrar a este club de privilegiados. El Tiempo [Economía]. Recuperado de http://www.eltiempo.com/economia/sectores/ asi-es-el-proceso-para-que-colombia-ingrese-a-la-ocde-enel-2018-139028

Gutiérrez, M. (2014, 19 de septiembre). Colombia debe cumplir 23 tareas para entrar a la OCDE. Portafolio [Finanzas]. Recuperado de http://www.portafolio.co/economia/finanzas/colombia-debe-cumplir-23-tareas-entrar-ocde- 48528

Merino, J. I. (2002). Impacto jurídico del ingreso de Chile a la OCDE. Anuario de Derecho Público UDP 2010(1), 319-329. Recuperado de http://derecho.udp.cl/wp-content/uploads/2016/08/Anuario_Derecho_Publico_2010. pdf

Nieto, J. A. (2011). España en la OCDE: Avances hacia el Estado de Bienestar. Revista de Economía Mundial, (28), 39-65.

OCDE. (2007). Relaciones con los medios de comunicación. Recuperado de http://www.oecd.org/centrodemexico/44358788. pdf

OCDE. (2017). Estudios económicos de la OCDE. Mejores politicas para una vida mejor. Recuperado de www.oecd.org/ eco/surveys/economic-survey-colombia.htm

Observatorio de la Cooperación Internacional - Colombia (2011). Colombia miembro pleno de la OCDE: ¿Un jugador de talla mundial? Bogotá, D.C. Recuperado de http:// studyres.es/doc/1721542/colombia-miembro-pleno-de-laocde--un-jugador-de-talla

Pradilla, A. (2012). Lineas directrices de la OCDE: La experiencia colombiana. Punto Nacional de Contacto de las Lineas Directrices de la OCDE para Empresas Multinacionales. Ministerio de Comercio, Industria y Turismo. Recuperado de http://trade.ec.europa.eu/doclib/docs/2012/ october/tradoc_150014.pdf 
República de Colombia. Congreso de la República. (4 de mayo del 2011). Por medio de la cual se escinden unos Ministerios, se otorgan precisas facultades extraordinarias al presidente de la República para modificar la estructura de la administración pública y la planta de personal de la Fiscalía General de la Nación y se dictan otras disposiciones. [Ley 1444]. DO: 48.059.

República de Colombia. Presidencia de la República. (2015). Proceso de acceso de Colombia a la OCDE. OCDE

República de Colombia. Presidencia de la República. Departamento Administrativo - DAPRE (15 de noviembre de 2013). Audiencia pública de rendición de cuentas del Departamento Administrativo de la Presidencia de la República. [Documento técnico]. Recuperado de http://es.presidencia.gov.co/oci/ DocumentosOCI/informe-congreso-dapre-2013.pdf

República de Colombia. Presidencia de la República. (2017). Colombia camino a la OECD. Buenas Prácticas Regulatorias. Recuperado de http://www.aecidcf.org.co/Ponencias/2017/marzo/MI160317-1/9.Buenas_Practicas_Regulatorias.pdf

Rojas, J. F. (Octubre de 2013). La dura tarea para ingresar al "club OCDE". El Colombiano [Economía]. pp. 18-19. Recuperado de http://www.elcolombiano.com/historico/la_dura_ tarea_para_ingresar_al_club_ocde-IYEC_266934

Sáez, R. E. (2010). La OCDE y el ingreso de Chile. Estudios Internacionales, (166), 93-112.

Universidad EAFIT. (17 de marzo de 2016). ¿En el "club de los países ricos"? Academia Escuela de Derecho/Noticias. Recuperado de http://www.eafit.edu.co/escuelas/derecho/ noticias/Paginas/colombia-ocde.aspx 
Luz Elena Mira Olano es investigadora junior en Colciencias. Decana de la Facultad de Derecho y Ciencias Forenses de la Institución Universitaria Tecnológico de Antioquia. Doctora en Ciencias Jurídicas; magíster en Derechos Humanos, Democracia y Derecho Internacional; especialista en Derecho Penal; investigadora y docente universitaria de pregrado y posgrado. https://orcid.org/0000-0001-8686-0519

Jahir Alexander Gutiérrez Ossa es investigador asociado en Colciencias. Miembro de la Comisión de Ordenamiento Territorial para el Departamento de Antioquia 2015 - 2017. PhD. en Administración Pública de la Atlantic International University. Honolulu, USA. Magíster en Desarrollo con énfasis regional y local de la Universidad Pontificia Bolivariana y economista industrial de la Universidad de Medellín, ambas en Medellín, Antioquia. https://orcid.org/0000-0001-8564-0397 